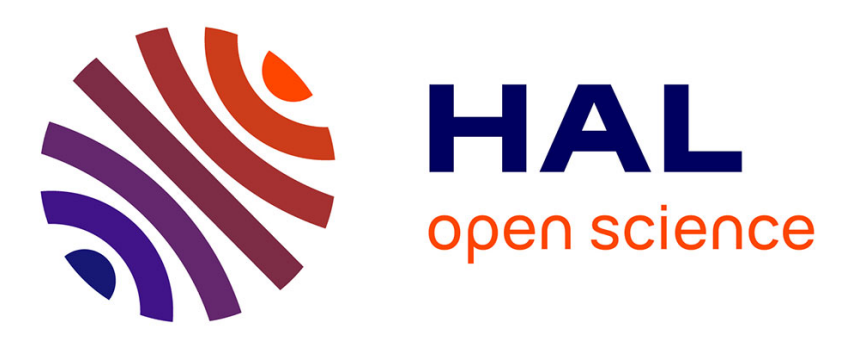

\title{
Transition from hydrodynamic turbulence to magnetohydrodynamic turbulence in von Kármán flows
}

Gautier Verhille, Ruslan Khalilov, Nicolas Plihon, Peter Frick, Jean-Francois Pinton

\section{- To cite this version:}

Gautier Verhille, Ruslan Khalilov, Nicolas Plihon, Peter Frick, Jean-Francois Pinton. Transition from hydrodynamic turbulence to magnetohydrodynamic turbulence in von Kármán flows. Journal of Fluid Mechanics, 2012, 693, pp.243-260. 10.1017/jfm.2011.522 . hal-00783162

\section{HAL Id: hal-00783162 https://hal.science/hal-00783162}

Submitted on 8 Dec 2019

HAL is a multi-disciplinary open access archive for the deposit and dissemination of scientific research documents, whether they are published or not. The documents may come from teaching and research institutions in France or abroad, or from public or private research centers.
L'archive ouverte pluridisciplinaire $\mathbf{H A L}$, est destinée au dépôt et à la diffusion de documents scientifiques de niveau recherche, publiés ou non, émanant des établissements d'enseignement et de recherche français ou étrangers, des laboratoires publics ou privés. 


\title{
Transition from hydrodynamic turbulence to magnetohydrodynamic turbulence in von Kármán flows
}

\author{
Gautier VERHILLE ${ }^{1,2}$, Ruslan KHALILOV ${ }^{3}$, Nicolas PLIHON ${ }^{1}$, \\ Peter FRICK ${ }^{3}$, Jean-François PINTON ${ }^{1}$
}

(1) Laboratoire de Physique, École Normale Supérieure de Lyon, CNRS UMR 5672 \& Université de Lyon, 46 allée d'Italie, F-69364 Lyon cedex 07, France

(2) Insitut de Recherche sur les Phénomènes Hors Équilibre, UMR 6594, CNRS \& Aix-Marseille Université, 49 rue F. Joliot-Curie, BP 146, 13384 Marseille cedex 13, France (3) Institute of Continuous Media Mechanics, Korolev 1, Perm 614013, Russia

(Received 2012)

The influence of an externally applied magnetic field on flow turbulence is investigated in liquid-gallium von Kármán (VK) swirling flows. Time-resolved measurements concern global variables (such as the flow power consumption) and local recordings of the induced magnetic field are made. From these measurements, an effective Reynolds number is introduced as $R m_{\mathrm{eff}}=R m(1-\alpha \sqrt{N})$, so as to take into account the influence of the interaction parameter $N$. This effective magnetic Reynolds number leads to unified scalings for both global variables and the locally induced magnetic field. In addition, when the flow rotation axis is perpendicular to the direction of the applied magnetic field, significant flow and induced magnetic field fluctuations are observed at low interaction parameter values, but corresponding to an Alfvèn speed $v_{A}$ of the order of the fluid velocity fluctuations $u_{\mathrm{rms}}$. This strong increase in the flow fluctuations is attributed to chaotic changes between hydrodynamic and magnetohydrodynamic velocity profiles.

\section{Introduction}

The understanding of how flows of electrically conducting fluids develop when a significant magnetic field is imposed is of both fundamental and technical importance. Such situations are common in practical applications such as electromagnetic pumping of conducting fluids, control of flow motions during metal casting or crystallization processes or confinement of thermonuclear plasmas in magnetic confinement devices. Magnetic fields are also ubiquitous in astrophysical bodies, such as galaxies, stars and planets, where the dynamo instability converts parts of the kinetic energy of flowing conducting fluids into magnetic energy. The influence of magnetic fields on astrophysical flows is thus also a generic feature (it can be observed for instance through the influence of the Earth's magnetosphere on the solar wind).

In the incompressible magnetohydrodynamics (MHD) approximation, the dynamics is governed by the coupled set of Navier-Stokes (NS) and induction equations:

$$
\begin{gathered}
\rho\left(\partial_{t} \mathbf{u}+(\mathbf{u} \cdot \nabla) \mathbf{u}\right)=-\nabla p+\rho \nu \Delta \mathbf{u}+\mathbf{j} \times \mathbf{B}, \\
\partial_{t} \mathbf{B}=\nabla \times(\mathbf{u} \times \mathbf{B})+\lambda \Delta \mathbf{B}, \\
\nabla \cdot \mathbf{u}=0
\end{gathered}
$$




$$
\nabla \cdot \mathbf{B}=0
$$

where $\mathbf{u}(\mathbf{r}, t), p(\mathbf{r}, t), \mathbf{B}(\mathbf{r}, t)$ are the velocity, pressure and magnetic fields in a fluid with (constant) density $\rho$, kinematic viscosity $\nu$ and magnetic diffusivity $\lambda=1 / \mu_{0} \sigma$, where $\sigma$ is the electrical conductivity of the fluid and $\mu_{0}$ the magnetic permeability; the current density stems from the Maxwell-Ampère equation, $\mu_{0} \mathbf{j}(\mathbf{r}, t)=\nabla \times \mathbf{B}(\mathbf{r}, t)$. The nature of the problem is usually governed by values of the main dimensionless parameters defined as a function of characteristic velocity $U$, length scale $L$ of the flow and typical value of the magnetic field $B_{0}$ from the analysis of the NS and induction equations. The kinetic Reynolds number $R e=U L / \nu$ compares the amplitude of the inertial term to the viscous term in the NS equation. In the limit of large kinetic Reynolds number one defines an interaction parameter $N=\sigma L B_{0}^{2} / \rho U$ which compares the Lorentz force to the inertial term in the N.S equation (in the low-Reynolds-number limit, the Hartmann number $H a^{2}=N R e$, - which compares the Lorentz force to the viscous term - is used). The magnetic Reynolds number $R m=U L / \lambda$ compares the induction term to the diffusion term in the induction equation. The ratio of the magnetic Reynolds number to the kinetic Reynolds number defines the magnetic Prandtl number $P m=\nu / \lambda$, which compares the diffusive temporal/length scales of the velocity and magnetic fields and depends only on the physical properties of the fluid.

Among the various situations in the $(R e, R m, N, P m)$ parameter space, some asymptotic limits have been studied in details. The case of flow with moderate kinetic Reynolds number and large interaction parameter number has been extensively studied for industrial applications. As explained and verified experimentally by Sommeria \& Moreau (1982), Moreau (1998) and Eckert et al. (2001), the Lorentz force acts as an anisotropic diffusivity which damps the velocity field fluctuations along the applied magnetic field and hence drives the flow toward a two-dimensional structure. The issue of exact twodimensional versus weak three-dimensional features has recently been addressed experimentally by Klein \& Pothérat (2010) and numerically by Mück et al. (2000). Another limit, relevant in several astrophysical situations, corresponds to large kinetic Reynolds numbers onto which a weak magnetic field is applied. In this case the interaction parameter is small, the Lorentz force does not influence significantly the hydrodynamics and the situation is reduced to the advection of a passive vector field by a (prescribed) turbulent velocity field. Several features have then been derived in the framework of the Kolmogorov theory of turbulence, as for instance the spatial spectrum of magnetic energy, $B^{2}(k) \propto k^{-11 / 3}$ - see for instance Moffatt (1961) - verified experimentally in Odier et al. (1998). An open question, currently debated, is the possibility that the flow turbulence induces an effective magnetic diffusivity which may become much larger than the molecular value, cf. Frick et al. (2010). The astrophysical limit of large values of the kinetic and magnetic Reynolds numbers has also received much attention during the last few decades. Among these studies, the understanding of the dynamo instability has benefited from joint theoretical, numerical and experimental efforts.

In the context of liquid-metal dynamos, especially relevant for planetary dynamo studies, a number of recent studies (Gailitis et al. 2001; Müller et al. 2006; Monchaux et al. 2009) explored a regime in the limit of large kinetic Reynolds numbers and moderate (to high) interaction parameter values. Dynamo action has so far been observed in three experiments using liquid-sodium as the working fluid: the Riga experiment, based on a Ponomarenko screw flow (Ponomarenko 1973); the Karlsruhe experiment using an array of like-sign helical motions as proposed by G.O. Roberts (Roberts 1972) ; and the von Kármán sodium (VKS) experiment based on swirling motions, as studied numerically by Dudley and James (Dudley \& James 1989). In liquid-sodium flows, owing to the very 
low value of the magnetic Prandtl number Pm, even moderate values of $R m$ are associated with very high $R e$ values, i.e. fully developed turbulence. In this context, how the self-generated dynamo field acts on the velocity field so as to saturate the growth of the (supercritical) instability is an open problem. In the Riga experiment, a significant reduction of the rotational velocity of the jet was observed, as shown in Gailitis et al. (2001) and Kenjeres \& Hanjalic (2009). In the same manner the dynamo field in the Karlsruhe experiment led to a measurable braking of the fluid in the pipe system (Müller et al. 2006). In the case of the VKS dynamo (Monchaux et al. 2009), the coupled (u, B) evolution has not yet been measured and the interplay between magnetic and velocity fields is yet to be clarified.

It is the purpose of the study presented here to investigate how a fully developed turbulent flow interacts with a strong applied magnetic field. Investigated flows are of von Kármán type, generated in a cylinder by the rotation of impellers, and hence of the same kind as the VKS dynamo flow, as reported for instance in Monchaux et al. (2009). Two applied magnetic field geometries have been studied: the applied magnetic field may be perpendicular or parallel to the flow rotation axis. Details of the experimental setup are given in section 2 . The variation of a global quantity, namely the power injected into the flow in order to keep the driving impellers at a fixed rotation rate, with the intensity of the applied magnetic field is then discussed in section 3. We show that it varies linearly with the interaction parameter $N$. This scaling is confirmed in section 4 by the analysis of the local induction measurements. In section 5, we detail a noteworthy finding of our study: in the transition from a purely hydrodynamic regime (for the lowest $N$ values) to a fully "magnetic" state (at high $N$, for which turbulence is damped), an increase in the flow fluctuations has been observed. This increase is mainly seen in the long-time dynamics of the flow, and was observed when the influence of the magnetic field balances the influence of the flow rotation.

\section{Experimental set-up}

The liquid-gallium flow is generated by the rotation of two impellers at each end of a cylindrical vessel. The vessel is sketched in figure 1 (a) and has a radius $R_{c}$ of $97 \mathrm{~mm}$ and a length of $323 \mathrm{~mm}$. The impellers have a diameter equal to $2 R=165 \mathrm{~mm}$ and are fitted to a set of eight straight blades with height $10 \mathrm{~mm}$. All parts used in this setup are machined from non-magnetic stainless-steel. The two impellers are separated by a distance $H=203 \mathrm{~mm}$. They are independently driven by two AC-motors which provide a constant rotation rate in the interval $\left(\left|F_{1}\right|,\left|F_{2}\right|\right) \in[0.5,25] \mathrm{Hz}$. When constrained to operate at constant rotation speed, the motors electric drives deliver an analogue output proportional to the current in the motor, as an image of the applied mechanical torque.

Liquid-gallium is cooled by water circulation located behind the driving impellers; the experiments are run at a constant temperature between $40{ }^{\circ} \mathrm{C}$ and $48^{\circ} \mathrm{C}$. Liquid gallium has density $\rho=6.09 \times 10^{3} \mathrm{~kg} \cdot \mathrm{m}^{-3}$, electrical conductivity $\sigma=3.68 \times 10^{6} \Omega^{-1} \cdot \mathrm{m}^{-1}$, hence a magnetic diffusivity $\lambda=1 / \mu_{0} \sigma=0.29 \mathrm{~m}^{2} \cdot \mathrm{s}^{-1}$. Its kinematic viscosity is $\nu=$ $3.1 \times 10^{-7} \mathrm{~m}^{2} \cdot \mathrm{s}^{-1}$. In the present study, the flow is driven either by both impellers rotating at equal speed, in the same or opposite direction, or by the rotation of only one disk. In any configuration, the integral kinematic and magnetic Reynolds numbers are defined as $R e=2 \pi R^{2} F / \nu$ and $R m=2 \pi R^{2} F / \lambda$ where $F$ is the rotation rate (in Hertz) and $R$ the radius of the driving impeller(s). $R m$ values up to 5 are achieved, with corresponding $R e$ in excess of $10^{6}$. The liquid-gallium flows generated are thus fully turbulent, with high levels of fluctuations, depending on the topology of the time-averaged flows. Detailed experimental investigations of time-averaged flow topologies, fluctuation levels 


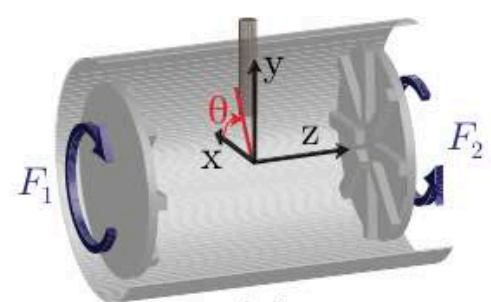

(a)

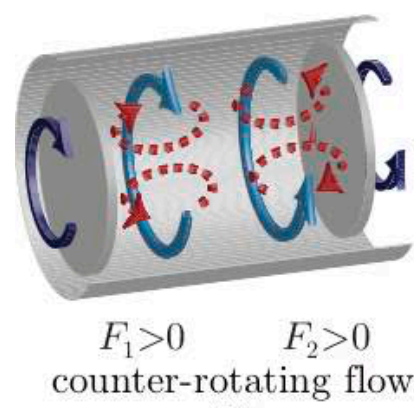

(b)

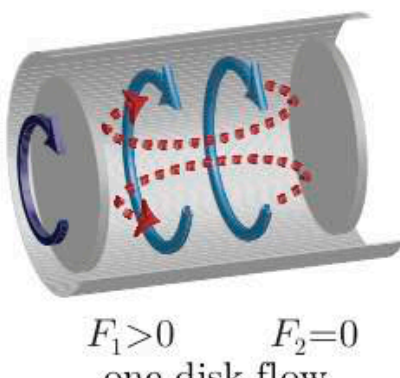

one-disk flow

(c)

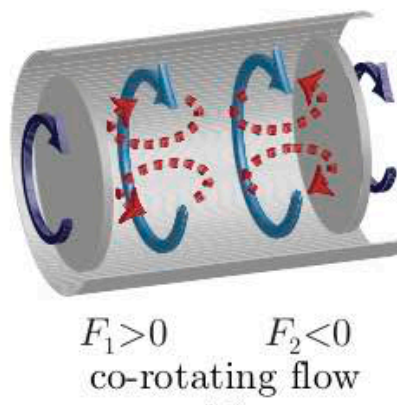

(d)

Figure 1. (a) Geometry of the experimental setup (see details in the text), and coordinate system used throughout the text; the magnetic probe shaft is along the $y$-axis. (b) Counter-rotating (s2t2) flow : two counter-rotating toroidal cells (solid arrows) and two poloidal cells (dashed arrows). (c) One-disk ( $s 1 t 1)$ flow : one toroidal cell and one poloidal cell. (d) Corotating ( $s 2 t 1)$ flow: one toroidal cell and two poloidal cells.

and characteristics may be found in (Ravelet et al. 2008).

Three flow topologies were considered in this study, depending on the relative values of $F_{1}$ and $F_{2}$. These flows are well documented from water experiments (Ravelet et al. 2008) and their time-averaged features are briefly recalled here. The 'counter-rotating' flow is defined as corresponding to the case of the two impellers rotating in opposite directions at the same speed (i.e. $F_{1}=F_{2}$ with the notation of figure $1(\mathrm{a})$ ). In this case, the mean flow is made up of two toroidal cells rotating in opposite directions and two poloidal cells due to the centrifugal forces localized close to the impellers, as sketched in figure 1(b). The 'counter-rotating' flow is a cylindrical equivalent of the s2t2 flow introduced by Dudley \& James (1989). Slow dynamics of the flow (as compared to the period of impellers' rotation) was shown to be linked to the evolution of the shear layer localized in the mid-plane in de la Torre \& Burguete (2007). If only one impeller rotates at the frequency $F$ and the other one is kept at rest, the mean flow is made up of one single toroidal cell and one poloidal cell as shown in figure 1(c). We refer to this flow topology as the "one-disk" flow which corresponds to the $s 1 t 1$ flow introduced by Dudley \& James (1989). Finally, when the two impellers rotate in the same direction and at the same rotation rate, the mean flow is made up of one toroidal cell and two poloidal cells, as shown in figure 1(d). This flow topology is referred to as the 'corotating' flow (i.e. $F_{1}=-F_{2}$ with the notation of figure 1(a)), and is of the s2t1 type introduced by Dudley \& James (1989).

A set of two water-cooled electrical coils can be arranged in an Helmholtz-like configuration to produce an applied magnetic field $B^{A}$ either aligned with the axis of the impellers (along the $z$-axis) or perpendicular to it (along the $x$-axis). The current in the coils is controlled and adjusted between 0 and 150 A by a Drusch $30 \mathrm{~kW}$ power supply. 


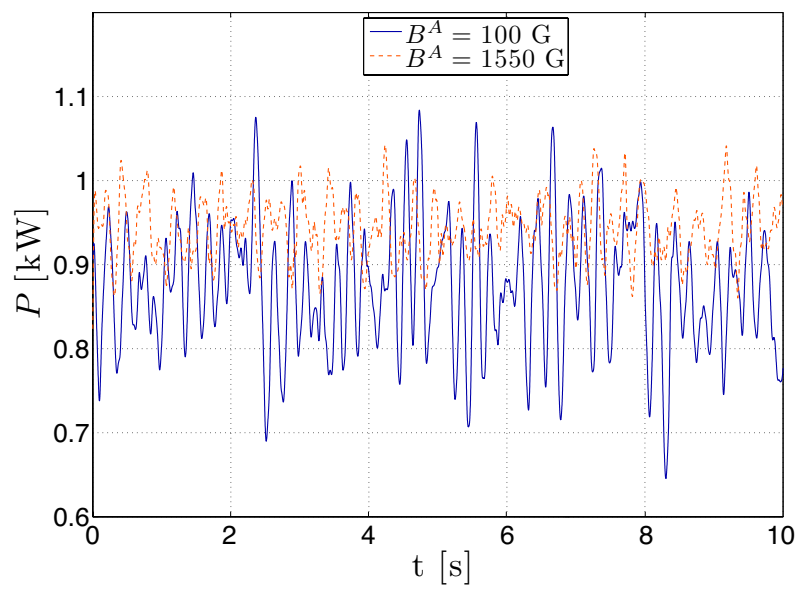

Figure 2. Time evolution of the power consumption, measured from the output of the motors' electric power supplies. Counter-rotating case at $10 \mathrm{~Hz}$ with an applied magnetic field $B^{A}=100 \mathrm{G}$ (solid line) and $B^{A}=1550 \mathrm{G}$ (dashed line).

The resulting applied magnetic field $B^{A}$ has an intensity which can reach up to $2000 \mathrm{G}$. Measurements show that the applied magnetic field is homogeneous within $10 \%$ over the vessel volume. As for the Reynolds numbers, an integral interaction parameter can be defined as $N=\sigma\left(B^{A}\right)^{2} / \rho 2 \pi F$. Its value ranges between 0 and 0.5 depending on the operational conditions. The maximal value of the interaction parameter is achieved for $F=2 \mathrm{~Hz}$. Below this rotation frequency the flow is not fully turbulent even in the absence of an externally applied magnetic field.

Magnetic measurements are obtained either with a homemade Hall-sensors array (Sentron CSA-1V), or with a commercial Bell Gaussmeter, both probing one component of the magnetic field. The bandwidth of these sensors is over $150 \mathrm{~Hz}$ so both time averages and fluctuations of the induced magnetic field $B^{I}$ are probed. The spatial evolution of the induced magnetic field is probed by an Hall sensor array at eight positions along a radius of the vessel. For every configuration in this study, the magnetic probes lie in the mid-plane at $\theta=\pi / 2$, i.e. parallel to the $y$-axis (see figure 1a). Magnetic field data and electrical signals from the motor drives are recorded using a National Instrument PXI-4472 digitizer (resolution of 23 bits) at a sampling rate equal to $1 \mathrm{kHz}$.

\section{Global behavior: injected power}

In this section, the influence of the applied magnetic field $B^{A}$ on the global power budget of turbulent flows is addressed. We note that, as torques and rotation rates of the impellers are conjugate variables, it is not possible to keep both at constant value. Thus, for each run, the rotation rates of the driving impellers are kept constant and the time evolutions of the driving torques are recorded. The time-averaged injected power is then computed as the product of the driving rotation rate times the time-averaged driving torque, summed for the two impellers. Figure 2 shows the time series for the injected power in the counter-rotating flow case, at $F=10 \mathrm{~Hz}$, for two values of the transverse applied magnetic field: $B^{A}=100 \mathrm{G}$ and $1550 \mathrm{G}$. As expected, under the increased action of Lorentz force, one clearly observes an increase of the mean power consumption and a decrease in the fluctuations.

Hereafter, we focus on the evolution of the mean power consumption. Let us first 
consider the hydrodynamic regimes, i.e. when $B^{A}=0$. The injected power grows as the third power of the rotation rate $F$ of the impellers, reflecting the turbulent nature of the flow. This scaling is indeed obtained in the limit of very large kinetic Reynolds numbers, when the flow power consumption is independent of the viscosity of the fluid. In turbulent flows, the power dissipated by the flow is equal to $P_{H}=M \epsilon$, where $M \simeq \rho R^{3}$ is the mass of fluid set in motion and $\epsilon=u_{\mathrm{rms}}^{3} / R$ is the injected power per unit mass in the turbulent cascade. The injected power is thus proportional to $R^{2} u_{r m s}^{3} \propto F^{3}$. In order to compare our measurements with previous studies, let us introduce a dimensionless power number (per motor) $K_{P}$, as $K_{P}=P_{H} /\left(2 \rho R^{5}(2 \pi F)^{3}\right)$, following Ravelet et al. (2005). Measured values are $K_{P}=10^{-2}$ for the corotating flow, $K_{P}=1.31 \times 10^{-2}$ for the one-disk flow, and $K_{P}=3.57 \times 10^{-2}$ for the counter-rotating flow. These differences are linked to the intensity of the large-scale shearing motions in the flow, and resulting velocity fluctuations - as shown by Marié \& Daviaud (2004). The measured $K_{P}$ are in close accordance with measurements in water flows in similar conditions (Ravelet 2005).

Let us now consider the case of a transverse applied magnetic field, i.e. the magnetic field is parallel to the $x$-axis and perpendicular to the axis of rotation of the impellers. (see figure 3). When increasing the intensity of the applied magnetic field, a first observation is that, in all configurations, the power injection at a constant rotation rate increases (cf. figure 3a-c). This increase can be ascribed to the Joule dissipation resulting from the induced electrical currents (or equivalently to the Lorentz force trying to slow down the flow); the total power dissipation results from viscous friction and Joule heating. However, the evolution of the injected power remains proportional to $F^{3}$ even for the highest applied magnetic field in each configuration. This suggests that the power consumption is still dominated by turbulent dissipation, with a modification of the large scale flow as detailed in section 5 .

For the three configurations, at first-order, the total injected power $P_{T}$ varies linearly with the interaction parameter $N$, as displayed in figure $3(\mathrm{~d}-\mathrm{f})$. This scaling will now be explained using dimensional analysis. As was pointed out previously, in the presence of a magnetic field, the nature of the problem involves a coupling between the velocity and the magnetic fields $(\mathbf{u}, \mathbf{B})$. In the remainder of the paper, the uncoupled hydrodynamic fields will be denoted $(U, 0)$ in the absence of an applied magnetic field, while the magnetohydrodynamic fields will be denoted $\left(U^{\prime}, B\right)$ in the presence of an applied magnetic field $B^{A}$ - with $B=B^{A}+B^{I}$. On average, the injected power in a turbulent flow is equal to that dissipated. The total dissipation $P_{T}$ in presence of an applied field is the sum of two terms: the turbulent dissipation $P_{H}\left(U^{\prime}, B\right)$ and the Joule dissipation $P_{J}\left(U^{\prime}, B\right)$ depending on both the velocity field and the magnetic field. As in the experiments the interaction parameter is moderate, one can assume, to first-order, that the turbulent dissipation is not modified by the Lorentz force:

$$
P_{H}\left(U^{\prime}, B\right) \sim P_{H}(U, 0) \sim \rho R^{2} u_{\mathrm{rms}}^{3}
$$

This assumption holds if the turbulent cascade is not significantly modified, as is confirmed by the spectra of the induced magnetic field presented later in section 5 and also as reported in Alemany et al. (1979). The Joule dissipation is defined as $P_{J}\left(U^{\prime}, B\right)=$ $\int_{\mathcal{V}} j^{2} / \sigma \mathrm{d} \tau \sim j^{2} L^{3} / \sigma, \mathcal{V}$ being the flow volume. As often observed in gallium flows (see for instance Bourgoin et al. (2004)), the induced magnetic field is small compared to the applied magnetic field $\left(B^{I} / B^{A} \sim 0.5\right)$ and, again in a first approximation, one assumes that the current density $j$ scales as $j \sim \sigma U^{\prime} B^{A}$. So the Joule dissipation can be written 

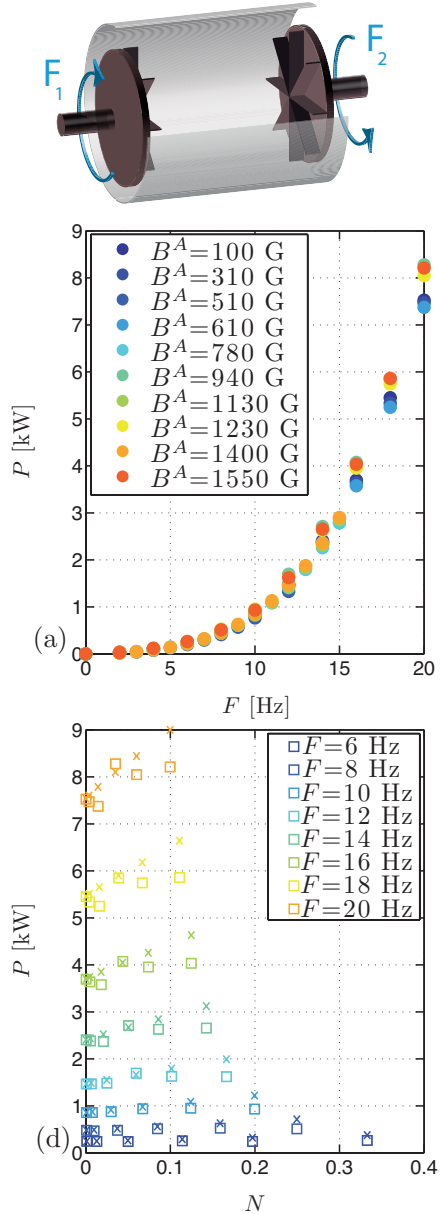
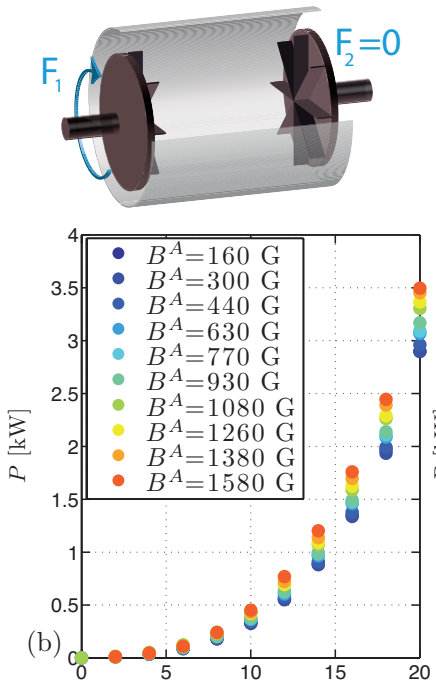

$F[\mathrm{~Hz}]$

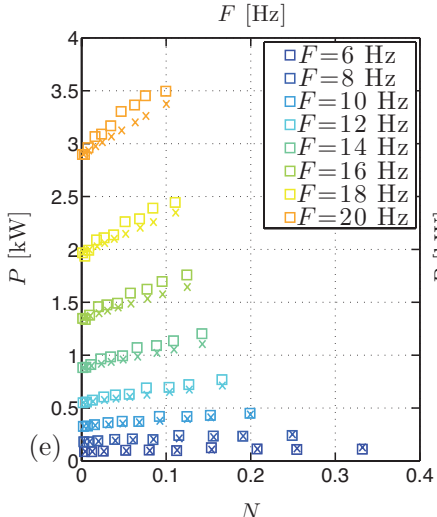

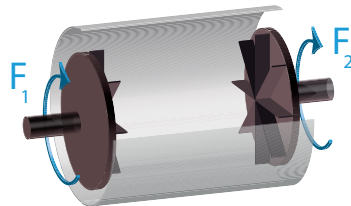
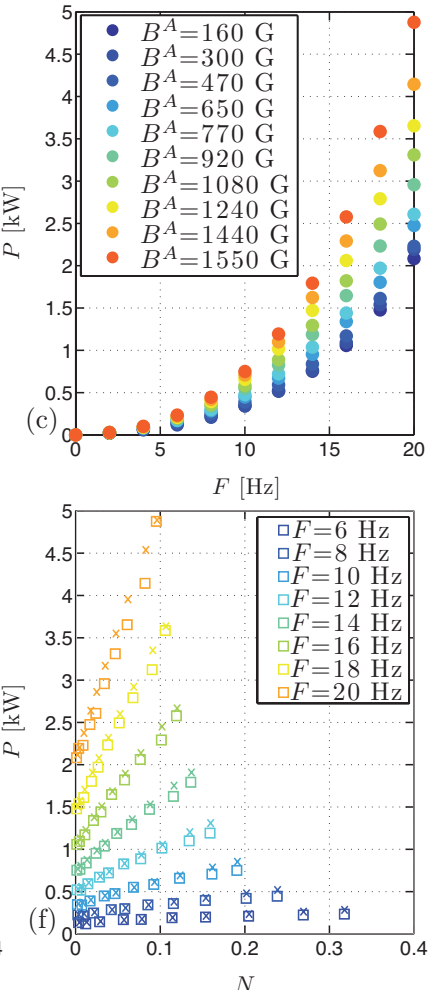

Figure 3. Evolution of the injected power as a function of the rotation rate $F$ (top panels (a) to (c)) and as a function of the interaction parameter $N$ (bottom panels (d) to (f)): measurements $(\square)$ and estimation $(x)$, see section 4.2. The applied magnetic field is perpendicular to the axis of rotation. The three investigated flows are: counter-rotating flow $(\mathrm{a}, \mathrm{d})$, one-disk flow $(\mathrm{b}, \mathrm{e})$ and corotating flow $(\mathrm{c}, \mathrm{f})$

as:

$$
P_{J}\left(U^{\prime}, B\right) \sim P_{J}\left(U^{\prime}, B^{A}\right) \sim \sigma U^{\prime 2}\left(B^{A}\right)^{2} R^{3} .
$$

At this order, one also assumes that the typical velocity (and the turbulent fluctuations) do not vary with $B^{A}$ - this approximation, certainly valid at low $N$ values, deteriorates as the applied magnetic field increases. So, by taking $U^{\prime}=u_{\mathrm{rms}}$, the Joule dissipation can be estimated as:

$$
P_{J}\left(U^{\prime}, B\right) \sim P_{J}\left(U, B^{A}\right) \sim \sigma u_{\mathrm{rms}}^{2}\left(B^{A}\right)^{2} R^{3}=\frac{\sigma\left(B^{A}\right)^{2} R}{\rho u_{\mathrm{rms}}} \cdot \rho u_{\mathrm{rms}}^{3} R^{2} \propto N P_{H}(U, 0),
$$

so that one expects, at first-order, the net power delivered by the motors to scale as

$$
P_{T}\left(U^{\prime}, B\right)=P_{H}(U, 0)+P_{J}\left(U, B^{A}\right)=P_{H}(U, 0)[1+\gamma N],
$$



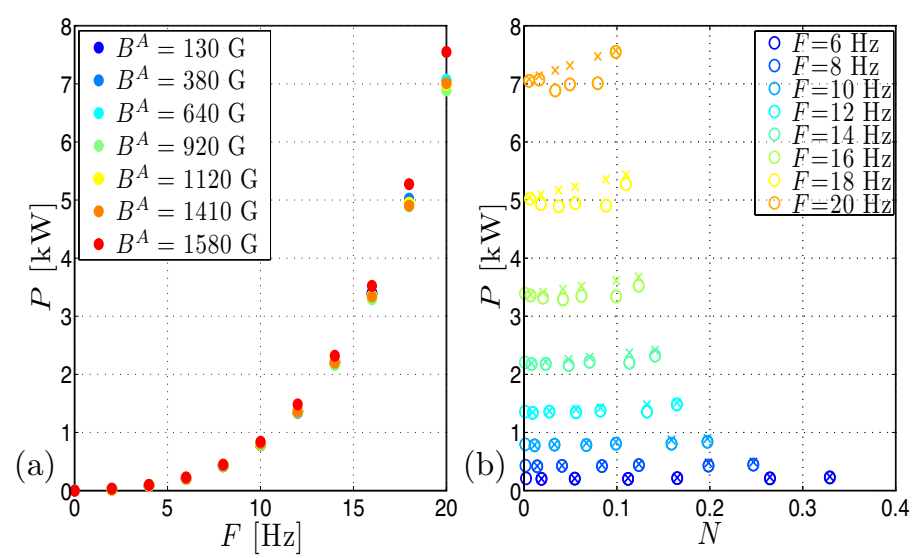

Figure 4. Evolution of the injected power for the counter-rotating flow with an axial applied field as a function of the rotation rate (a) and the interaction parameter (b): measurements (o), estimation $(x)$.

where $\gamma$ is a dimensionless constant which depends on the configuration. Figure 3(d-f) shows that, at first-order, this scaling is in good agreement with the measurements over the range of explored magnetic field intensities. However, note that the coefficient $\gamma$ is not constant as dimensionally predicted, but grows non linearly with the rotation rate of the impellers. A saturation of the power injection is also observed for the one-disk configuration. This traces back to our assumption that the net magnetic field in the flow is essentially the applied one. If, in the estimation of the induced current, one includes the induced magnetic field $B^{I}, j \sim \sigma U\left(B^{A}+B^{I}\right)$, with $B^{I}=\mathcal{F}\left(U, B^{A}\right)$, one introduces a dependence of $\gamma$ on $R m$ and $N$.

Let us now finally consider the case where the applied magnetic field is parallel to the axis of rotation, the two disks rotating in opposite directions and at the same speed. The induction processes with an axial applied magnetic field for other types of flow are much weaker and have not been systematically studied. The evolution of the injected power as a function of the impeller rotation rate, for increasing values of the applied magnetic field, is displayed in figure 4(a). As for a transverse applied magnetic field, the evolution of the injected power is not strongly affected by the amplitude of the applied magnetic field. Here the evolution as a function of the interaction parameter (figure 4(b)) is not linear: for small interaction parameter, the injected power decreases as the applied magnetic field increases; whereas for the highest interaction parameters, the power increases with the applied magnetic field. In this configuration, it is known that a strong applied magnetic field tends to decrease the Kelvin-Helmholtz instability which occurs in the mid-plane of a von Kármán flow (see for instance Biskamp (2003)). This laminarization of the shear layer tends to decrease the turbulent energy dissipated in the flow. This effect might be stronger than the dissipation linked to the Joule effect for low interaction parameter and thus lead to a decrease of the total injected power at the lowest $N$ values.

To conclude this section, let us return to our interpretation of the linear scaling of $P(N)$. At first-order, this argument, based on dimensional analysis, is valid as long as the energy transfer in the turbulent cascade scales as $u_{\mathrm{rms}}^{3} / \ell$. However, this simple argument is not sufficient to fully understand the evolution of the injected power which is strongly linked to the topology and the dynamics of the flow. Thus, this global analysis has to be supplemented by an inspection of the evolution of the local velocity gradients. For low- 
$R m$ flows, magnetic measurements provide this kind of information, as detailed in Volk et al. (2006).

\section{Local dynamics : induced magnetic field}

4.1. Experimental results

The evolution of a local variable, namely the induced magnetic field $B^{I}$, as a function of the interaction parameter $N$ is investigated for the different configurations studied. In this section, all measurements are performed in the mid-plane, at $r \sim 0.36 R$ along the $y$-axis.

As for the global variable, let us first consider the configurations with a transverse applied magnetic field. Before discussing the effect of large $N$ values on the induced magnetic field, the basic features of the time-averaged induction processes at low $N$ values are briefly recalled here for the three flows. For the counter-rotating flow, the main induced component at the measurement location is along the $z$-axis. This induction process is linked to the differential rotation of the toroidal cells and the electrical boundary condition of the flow, as described in Bourgoin et al. (2004). The induced magnetic field, normalized by the value of the applied magnetic field, was shown to vary linearly with $R \mathrm{~m}$. For the one-disk flow, the flow is strongly helical, and the induced magnetic field in the axial direction is generated by Parker's stretch-and-twist mechanism introduced in Parker (1955); the normalized induced magnetic field varies quadratically with $R m$ at low $N$ values, as observed experimentally in Pétrélis et al. (2003). Finally in the case of the corotating flow, the strong coherent vortex generated in the center of the flow vessel causes expulsion of the transverse magnetic field, as detailed in Moffatt (1978); this effect is tracked by measurements of the radial component of the induced magnetic field (Odier et al. 2000); the normalized induced magnetic field varies linearly with $R m$ at small $R m$ values, before showing saturation when the magnetic expulsion from the vortex becomes significant.

The evolution of the time-averaged value of the induced magnetic field, normalized by the value of the applied magnetic field, is displayed in figure $5(\mathrm{a}-\mathrm{c})$ as a function of the magnetic Reynolds number, for increasing values of the applied magnetic field. For small values of the applied magnetic field, the normalized induced magnetic field varies with the magnetic Reynolds number as described above. The situation changes significantly as the intensity of the applied magnetic field is increased. The amplitude of the induced magnetic field decreases for all flows, and the induction mechanisms become more complex, as illustrated in the case of the counter-rotating flow: the evolution with $R m$ is no longer linear and changes noticeably with $B^{A}$ (note in figure 5 (a) the significant changes even for quite moderate values of the applied magnetic field).

However, it will now be shown that measurements collapse onto a master curve for each configuration. In a previous study of the influence of large magnetic field on turbulent flows, Brito et al. (1995) have shown that a unified behavior can be derived when using the (modified) value of the flow velocity in the definition of an effective magnetic Reynolds number. This is indeed what is observed in the present case when using $R m_{\text {eff }}=R m(1-\alpha \sqrt{N})$, where $\alpha$ is a dimensionless constant which depends only on the configuration. The discussion on the derivation of such an effective magnetic Reynolds is left to subsection 4.2. The corresponding rescaled curves are shown in the bottom panels of figure 5(d-f) with $\alpha=3.8$ for the counter-rotating flow, $\alpha=0.7$ for the one-disk flow and $\alpha=1.15$ for the corotating flow. For each configuration, the collapse is obtained with one adjustable parameter, the coefficient $\alpha$, which does not depend on the amplitude of 
10
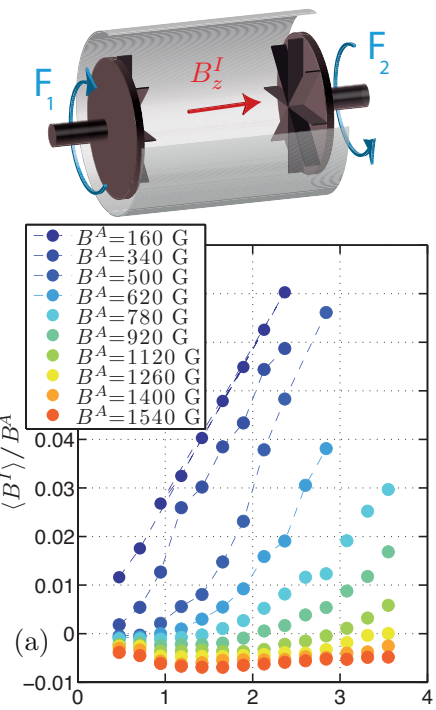

$R m$

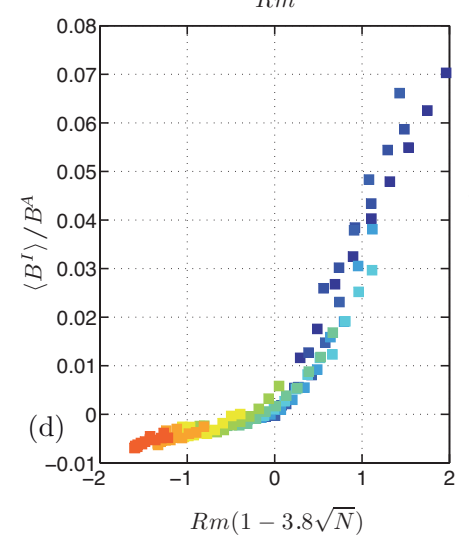

G. VERHILLE et al.
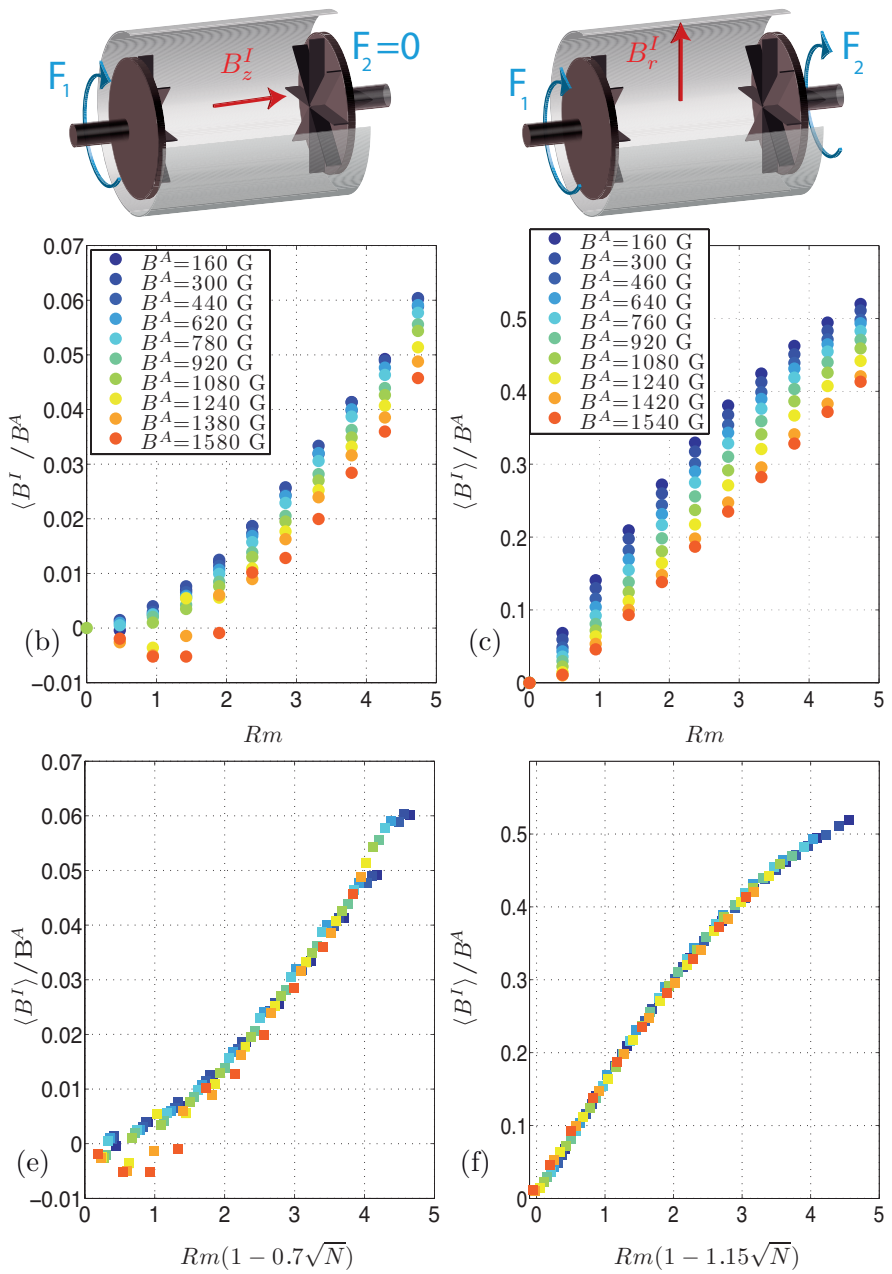

FIGURE 5. Evolution of the induced magnetic field $B^{I}$ (straight arrows as shown in the sketch of the experimental setup in figure 1) as a function of the magnetic Reynolds number $F$ for increasing values of the transverse applied magnetic field $B^{A}(\mathrm{a}-\mathrm{c})$, and as a function of an effective magnetic Reynolds number $\operatorname{Rm}(1-\alpha \sqrt{N})$ (d-f). Three distinct induction processes are probed for the three investigated flows (see text for details): counter-rotating flow (a-d), one-disk flow (b-e) and corotating flow (c-f).

$B^{A}$.

Let us now consider the configuration with an applied magnetic parallel to the axis of rotation of the impellers, with the counter-rotating flow. The leading-order induction process, at the measurement location, is the so-called $\omega$-effect, which induces an azimuthal magnetic field from the strong differential velocity of the toroidal cells (Bourgoin et al. 2004); at low $N$ values, the normalized induced magnetic field varies linearly with $R m$. Its evolution as a function of the magnetic Reynolds number is displayed in figure 6(a) for increasing values of the applied magnetic field. Similarly to the transverse configurations, the induced magnetic field is reduced at large $N$ values, and the effective magnetic Reynolds number $R m_{\mathrm{eff}} \propto R m(1-\alpha \sqrt{N})$ is also observed to collapse the data, as shown in figure $6(\mathrm{~b})$ with $\alpha=0.5$. 

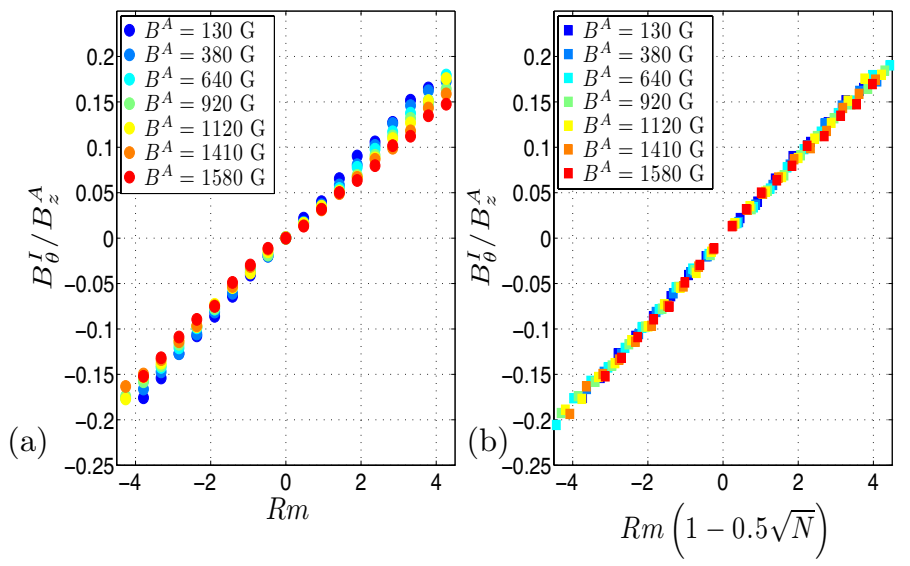

FIGURE 6. Evolution of the azimuthal induced magnetic field from an axial applied magnetic field with a counter-rotating flow, as a function (a) of the magnetic Reynolds number $\mathrm{Rm}$ and (b) of an effective magnetic Reynolds number $\operatorname{Rm}(1-\alpha \sqrt{N})$.

A similar study in the axial configuration has been reported in Gallet et al. (2009). In that paper, it is shown that the induced magnetic field $B^{I}$ normalized by $R m B^{A}$ only depends on the interaction parameter $N$. It is possible to fit these data using the scaling we propose with $\alpha=0.56$, which is close to the value measured in the present work. A direct comparison of the behavior of the small-scale fluctuations is less straightforward. Gallet et al. (2009) report velocity fluctuations using a bandpass potential probe and observed that the spectrum is mainly modified at high frequencies, i.e. for turbulent scales. In our case, the spectrum of the induced magnetic field reported in section 5 shows that the dominant effect of the magnetic field lies in the dynamics of the largescales.

\subsection{Discussion and estimation of the Joule dissipation}

In this subsection, based on power budget estimates, we first give an explanation for the expression of the effective magnetic Reynolds number, $R m_{\mathrm{eff}}=R m(1-\alpha \sqrt{N})$, then we discuss the consistency of our approach by evaluating the Joule dissipation in the system.

Let us now return to the flow power consumption, $P_{T}\left(U^{\prime}, B\right)=P_{H}\left(U^{\prime}, B\right)+P_{J}\left(U^{\prime}, B\right)$ addressed in section 3. Since no direct measure of the changes in the velocity field as the applied magnetic field is imposed is available, and since the velocity field and the magnetic field are coupled, any approach to determine the relative importance of the Joule dissipation compared to the viscous dissipation is based on a series of approximations. In section 3, the lowest order was considered, for which (i) the induced magnetic field is neglected in the Joule dissipation and (ii) the Lorentz force is neglected and the hydrodynamic dissipation is estimated assuming an unperturbed velocity field. In that case we showed that $P_{J}\left(U, B^{A}\right) \propto N P_{H}(U, 0)$ and that

$$
P_{T}\left(U^{\prime}, B\right) \sim P_{T}\left(U, B^{A}\right)=P_{H}(U, 0)[1+\gamma N]
$$

which is in good agreement with the power consumption data, but which also implies that $B^{I} / B^{A} \propto R m$. The ratio of the induced magnetic field to the applied magnetic field should scale linearly with $R m$, which is in contradiction with our observations. To account for the observed evolution of $B^{I} / B^{A}$ with $R m$ and $N$ and its rescaling using the 
effective magnetic Reynolds number $\operatorname{Rm}(1-\alpha \sqrt{N})$, the next-order approximation should be considered: the modification of the velocity field under the action of the Lorentz force has to be estimated. As the interaction parameter is moderate, one can assume that the modification of velocity field $\mathbf{v}$ by the Lorentz force is small: $\mathbf{v}=\mathbf{u}^{\prime}-\mathbf{u} \ll \mathbf{u}$ where $\mathbf{u}^{\prime}$ and $\mathbf{u}$ are the velocity fields with and without an applied magnetic field. Let us now assume that (i) the induced field is neglected in the Joule dissipation as in section 3 , and (ii) the hydrodynamic dissipation is estimated from the modified velocity field $U^{\prime}$ :

$$
P_{T}\left(U^{\prime}, B\right) \sim P_{T}\left(U^{\prime}, B^{A}\right)=P_{H}(U, 0)+P_{H}(V, 0)+P_{J}\left(U, B^{A}\right)
$$

which implies that $\mathbf{u}$ and $\mathbf{v}$ are not correlated. The observed linearity of the injected power as a function of the interaction parameter suggests that the hydrodynamic dissipation linked to the velocity field $\mathbf{v}$ also scales linearly with $N: P_{H}(V, 0) \propto N P_{H}(U, 0)$. Writing $P_{H}(V, 0)$ as $\rho R^{3} V^{2} / \tau$ with $\tau$ the characteristic energy transfer time still being of the order of $U / R$, one gets

$$
P_{H}(V, 0) \sim \rho R^{2} V^{2} U \propto N P_{H}=N \rho R^{2} U^{3}
$$

The velocity perturbation is thus estimated as $V \propto U \sqrt{N}$. Using the modified velocity $\mathrm{U}^{\prime}=\mathrm{U}+\mathrm{V}$ in the estimation of an effective magnetic Reynolds number as was introduced in Brito et al. (1995), indeed leads to an effective magnetic Reynolds number defined as $R m(1-\alpha \sqrt{N})$, as proposed in section 4.1 .

Finally, as a complementary approach and to the same order of approximation as for equation 4.2, one can assume that (i) the induced magnetic field has to be taken into account for the Joule power dissipation and (ii) that the Lorentz force is neglected:

$$
P_{T}\left(U^{\prime}, B\right) \sim P_{T}(U, B)=P_{H}(U, 0)+P_{J}\left(U, B^{A}+B^{I}\right)
$$

The magnetic induction measurements are now used to improve the estimation of the Joule power dissipation. For each flow configuration, the experimental curves $B^{I} / B^{A}$ are fitted by a function $g\left(R m_{\text {eff }}\right)$ with $R m_{\text {eff }}=R m(1-\alpha \sqrt{N})$. Joule dissipation is then expressed as

$$
P_{J}\left(U, B^{A}+B^{I}\right) \sim \sigma U^{2}\left(B^{I}+B^{A}\right)^{2} R^{3}=C_{J} N P_{H}\left(1+g\left(R m_{\mathrm{eff}}\right)\right)^{2}
$$

where $C_{J}$ is a dimensionless constant. The net power consumption can then be computed according to equation 4.4. These estimations are displayed as crosses $(\times)$ on figures 3 and 4 . Good agreement is found for $C_{J}=(1 / 2)^{3}$ for the one-disk flow and the counter-rotating flow with a transverse applied magnetic field, and $C_{J}=(1 / 3)^{3}$ for other configurations (this may be thought of as a change in the spatial extent of the electrical currents). The discrepancies for the largest $\mathrm{N}$ values (10-20\% of the injected power) may be due to the dissipation associated with the dynamics of the large scale.

To conclude this section, an investigation of the evolution of the induced magnetic field with the interaction parameter shows that the use of an effective magnetic Reynolds number $R m_{\text {eff }} \propto R m(1-\alpha \sqrt{N})$ is compatible with the global power dissipation scalings derived in section 3 and collapses the measurements for all investigated configurations. Although the power dissipation scaling is based on the assumption that the flow is only marginally modified, magnetic induction measurements show that the details of the flow topology can be locally strongly modified by the Lorentz force (refer to the dramatic modification of the induced magnetic field in figure 5(a)). A direct measurement of the velocity field is not yet available. However, a detailed study on the evolution of the 
induced magnetic field in the counter-rotating case as a function of $N$ would illustrate the action of the Lorentz force on the flow.

\section{Transition from hydrodynamic to magnetohydrodynamic flows}

We investigate in this section the details of how the transition from a situation where the magnetic fields plays the role of a passive vector to a situation where the magnetic field has a leading influence on the flow dynamics. In this problem, geometry is of prime importance. We will focus on the configuration where the applied magnetic field is perpendicular to the rotation axis of the counter-rotating flow of the von-Kármán flow. The rationale is that very large magnetic fields or rotation rates will each act to two-dimensionalize the flow in planes perpendicular to its direction. This competition is expected to, and does, generate non trivial dynamics (Zikanov \& Thess 1998), as presented here.

As in the previous section, all magnetic measurements are made in the mid-plane, at $r \sim 0.36 R$ along the $y$-axis, the applied magnetic field is parallel to the $x$-axis and the two impellers generate a counter-rotating flow by rotating at $F=12 \mathrm{~Hz}$ in opposite directions. As we want to study the transition, we limit ourselves to interaction parameter smaller than $0.1\left(B^{A} \sim 1200 \mathrm{G}\right)$. In the range $0.1<N<0.27$, no flow modification has been observed.

Figure 7(a) shows the evolution of the time-averaged induced magnetic field as a function of the interaction parameter. For small applied magnetic field, the induced magnetic field is proportional to the square root of the interaction parameter, which is equivalent to the induced magnetic field being proportional to the applied magnetic field, as expected at low $R m$, for which the induction equation is dominated by the balance between the stretching of field lines and diffusion, i.e. $\lambda \Delta \mathbf{B}^{I} \sim \mathbf{B}^{A} \cdot \nabla \mathbf{u}$. We refer to this regime as an "hydrodynamic" regime, because the main features are accounted for when one computes the induced magnetic field from an purely hydrodynamic velocity field (i.e. with vanishing influence of the Lorentz force in the NS equation). At higher magnetic fields, the $\sqrt{N}$ scaling is no longer observed, and the induced magnetic field eventually decreases with increasing $N$ values. This regime where the flow is strongly influenced by the magnetic field will be referred to as a magnetohydrodynamic (MHD) regime. The transition to this regime can be estimated in the following manner. From induction measurements, let us now redefine an effective magnetic Reynolds number as $R m_{\mathrm{eff}}^{\mathrm{ind}} \equiv B^{I} / B^{A}$. Then, our experimental observation is that the MHD regime is reached when $N>R m_{\mathrm{eff}}^{\text {ind }}$, as shown in figure $7(\mathrm{a})$. Physical insight is gained when expressing the interaction parameter as

$$
N=\frac{\sigma R B^{A^{2}}}{\rho u_{\mathrm{rms}}}=\mu \sigma R u_{\mathrm{rms}} \cdot \frac{B^{A^{2}}}{\rho \mu u_{\mathrm{rms}}^{2}} \sim R m_{\mathrm{eff}}^{\mathrm{ind}} \cdot\left(\frac{v_{A}}{u_{\mathrm{rms}}}\right)^{2},
$$

where $v_{A}$ is the Alfvèn velocity. In this context, the transition to the MHD regime happens when the Alfvèn velocity becomes of the order of the hydrodynamics velocity fluctuations (i.e. $N \sim R m_{\mathrm{eff}}^{\text {ind }}$ ). This is indeed observed in figure 7.a for $N \simeq 0.02$. For instance, for $F=12 \mathrm{~Hz}$, the maximal velocity is of the order of $U=2 \pi R F \sim 7 \mathrm{~m} / \mathrm{s}$ with fluctuations of about half that value (in the pure hydrodynamic situation); the Alfvèn speed for $B^{A}=1 \mathrm{kG}$ is $v_{A} \sim 1 \mathrm{~m} / \mathrm{s}$. The flow fluctuations locally excite Alfvèn waves which are evanescent in liquid metals (in conditions where the applied magnetic field is about $1 \mathrm{kG}$ ). The Joule dissipation linked to the local current is quite effective at damping the motion.

The fluctuations of the induced magnetic field display the same behavior as shown 

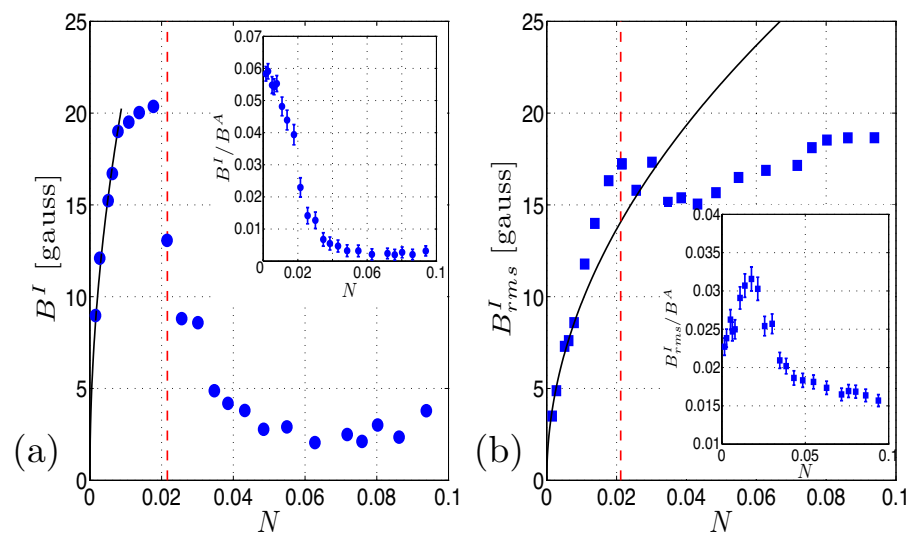

Figure 7. Evolution of the axial component of the induced magnetic field measured in the mid-plane as a function of $N$ : (a) time-averaged value; (b) standard deviation. The disks rotate at $12 \mathrm{~Hz}$ in opposite directions and the applied magnetic field is transverse. Insets show value normalized by the applied magnetic field. The solid line corresponds to a fit of the experimental measurements in the hydrodynamic regime and the dashed line corresponds to $N=B^{I} / B^{A}$.
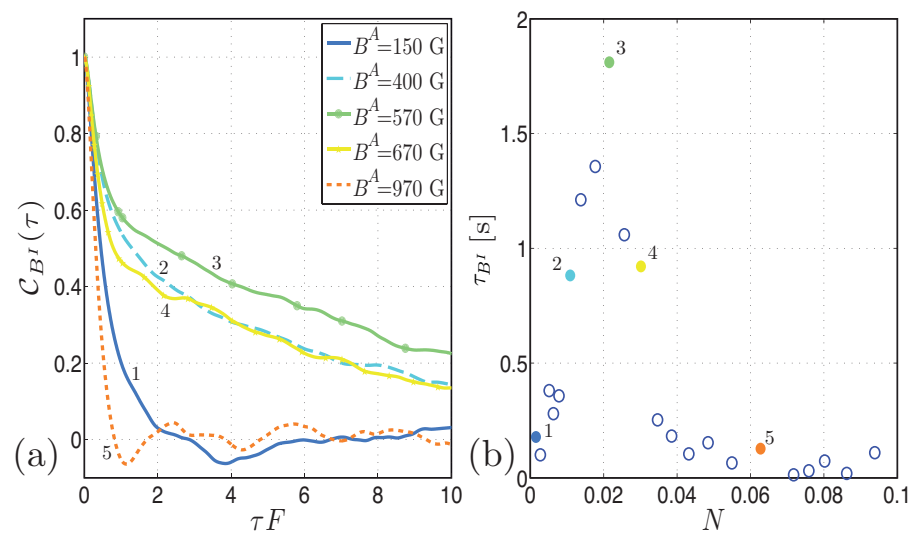

Figure 8. (a) Correlation function $\mathcal{C}_{B^{I}}(\tau)$ of the induced magnetic field for different applied magnetic fields. (b) Correlation time of the induced magnetic field as a function of the interaction parameter. Point with number correspond to values at which the correlation function evolution is displayed in (a).

in figure 7(b). The fluctuations normalized by the applied field $B^{A}$ also have a maximum for $N \simeq 0.02$ which corresponds to the transition observed in figure $7(\mathrm{a})$. The existence of a maximum suggests that the topology of the flow has changed, otherwise the decrease of the fluctuation amplitude would be monotonic. Another indication of topological changes is obtained by an analysis of the autocorrelation functions, $\mathcal{C}_{B^{I}}(\tau)=\left\langle B^{I}(t+\tau) B^{I}(t)\right\rangle_{t} /\left(B^{I} \mathrm{rms}\right)^{2}$. As seen in figure 8 , the correlation time, $\tau_{\mathcal{B}^{\mathcal{I}}}=$ $\int_{-\infty}^{\infty} \mathcal{C}_{B^{I}}(\tau) \mathrm{d} \tau$, is about 10 times longer in the transition regime than in the hydrodynamic regime or the MHD regime.

In order to emphasize the fact that changes in the transition regime correspond to slow changes, we analyze in figure 9(a) the power spectral density (PSD) of the induced magnetic field for three values of the applied magnetic field: $B^{A}=150 \mathrm{G}(N=1.5 \times$ 

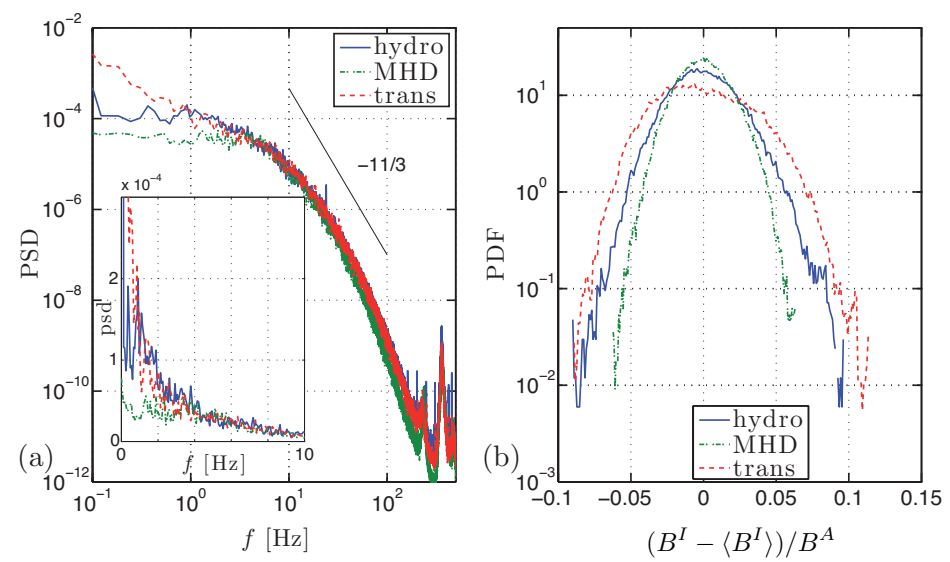

Figure 9. (a) Power spectral density of the normalized induced magnetic field with a transverse applied magnetic field and a counter-rotating flow, $F=12 \mathrm{~Hz}$, for three values of the applied magnetic field: $N=1.5 \times 10^{-3}, B^{A}=150 \mathrm{G}$, (blue); $N=1.4 \times 10^{-2}, B^{A}=460 \mathrm{G}$ (red) and $N=6.2 \times 10^{-2}, B^{A}=970 \mathrm{G}$ (green). (b) Corresponding centered probability density functions.

$\left.10^{-3}\right)$, i.e. in the hydrodynamic regime, $B^{A}=460 \mathrm{G}\left(N=1.4 \times 10^{-2}\right)$, in the transition region, and $B^{A}=970 \mathrm{G}\left(N=6.2 \times 10^{-2}\right)$ in the MHD regime. Comparison of the power spectral density of the signals shows that differences are clearly observed only in the low frequencies, while the turbulent inertial range is unmodified. The low-frequency dynamics of the induced magnetic field traces back to the large scale dynamics of the velocity field (de la Torre \& Burguete 2007). In the hydrodynamic regime, the lowfrequency spectral index of the induced magnetic field PSD is around $-1 / 2$ (Volk et al. 2006). In the transition regime, the increase of the low-frequency fluctuations lead to a spectral index reaching -1 . In the MHD regime, there is a dramatic damping of lowfrequency fluctuations. On the other hand, the high-frequency turbulent fluctuations are not modified at large applied magnetic field. For the three regimes, the spectral slope index is compatible with the turbulent $f^{-11 / 3}$ scaling.

The probability density functions (p.d.f.), displayed in figure 9(b), are also strongly modified when increasing the interaction parameter. The variance and the flatness are increased in the transition regime, before decreasing at larger values of the interaction parameter. For the three regimes, the deviation from a Gaussian disappears when highpass filtering the signal (cut-off frequency $f_{c}=F=12 \mathrm{~Hz}$ - not shown).

Such long correlation times and slow evolutions in turbulent flows have been previously reported in situations where the flow topology changes slowly in time between several preferential states (Cortet et al. 2010). As an attempt to test further this possibility of preferential states, we use an array of Hall probes to record the induced magnetic field profile along a radial direction in the vessel, as introduced in Volk et al. (2006). For the three previous values of the applied magnetic field $B^{A}$, we show in figure 10 the spatial evolution of the normalized magnetic field. The time-averaged spatial profile is displayed by filled black symbols embedded in a grey area which represents the standard deviation variations around the mean profile. Extrema are displayed by open and grey symbol for the furthest below and above the mean profile. The mean profile is defined as the time-averaged profile over 180 seconds, i.e. 1800 flow integral turnover times, which corresponds to 1300 magnetic diffusion times. Averages over $1 \mathrm{~s}$ are referred to as instantaneous profiles (10 turn-over times or 7 magnetic diffusion time).

In the hydrodynamic regime, the induction profile is identical to previous measure- 

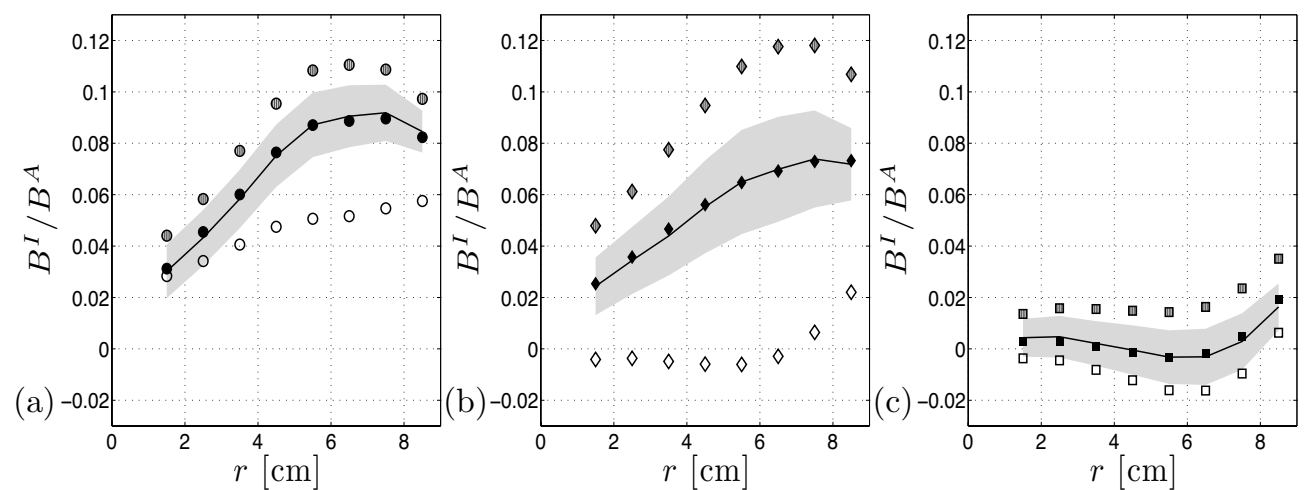

FIgURE 10. Instantaneous profiles of the axial induced magnetic field in the mid-plane: a) $B^{A}=150 \mathrm{G}$, hydrodynamic regime, b) $B^{A}=520 \mathrm{G}$, transitory regime, c) $B^{A}=1200 \mathrm{G}$, MHD regime. The black symbols correspond to the time averaged induction profile. The furthest profiles from the mean values are represented by dashed symbols (greater than the mean) and empty symbols (smaller than the mean). The gray area corresponds to the standard deviation of the profile.

ments by (Volk et al. 2006), which peaks at about $3 R / 4$ in the mid-plane. In the MHD regime, the shape is drastically different: the induced magnetic field no longer varies appreciably in the radial direction. In the transition regime, the induced profile is observed to change chaotically between a configuration which lies close the hydrodynamics case and a configuration close to the MHD case. The long-time dynamics of the transitions, the existence of hysteresis and the nature of the bifurcation between these two regimes are currently under investigation.

\section{Concluding remarks}

Our study has focused on essential effects of a magnetic field applied on a turbulent flow, as it evolves from a situation for which the magnetic field is a passive tracer to a situation where the Lorentz force is dominant. We have observed that, at leading order, the flow power consumption increases linearly with the interaction parameter $N$. A firstorder of approximation for the power consumption leads to the introduction of an effective magnetic Reynolds number $R m_{\text {eff }}=R m(1-\alpha \sqrt{N)}$. The effective magnetic Reynolds number, introduced from global measurements, also collapses local magnetic induction measurements. Finally, the very good agreement between the estimate of the Joule dissipation from induced magnetic field measurements at the same order of approximation and the measured power consumption showed the consistency of our approach. When the magnetic field is perpendicular to the axis of rotation of the VK flow, a refined study on the modification of the induced magnetic field at a given flow driving exhibits a transition from a hydrodynamic regime to an MHD regime as $N$ exceeds about 0.05 . In the MHD regime the large scale dynamics is significantly changed, essentially corresponding to a slow down of the flow under the action of the Lorentz force. The small-scale turbulent fluctuations are not significantly modified; this happens at larger values of the interaction parameter. In the transition regime from the hydrodynamic to the MHD regimes, strong increase in the flow fluctuations was observed. This corresponds to topological changes as the flow alternates chaotically between the original hydrodynamic mean flow profile and one dominated by MHD forces. This type of increased intermittency in the largescales may be generic in situations where the large scale vorticity and magnetic fields 
are perpendicular. Solving the details of this complex MHD dynamics requires highresolution measurements of the evolution of the local velocity field; such measurements are progressively becoming available, and will be implemented in future studies.

\section{Acknowledgements}

We acknowledge the technical support of M. Moulin. We thank an anonymous referee for remarks that raised the discussion of section 4.2. This work was supported by contract ANR-08-BLAN-0039-02 and CNRS-RFBR PICS Grant No. 07-01-92160

\section{REFERENCES}

Alemany, A., Moreau, R., Sulem, P.L. \& Frisch, U. 1979 Influence of an external magnetic field on homogeneous MHD turbulence. Journal de Mécanique 18 (2), 277-313.

Biskamp, D. 2003 Magnetohydrodynamic turbulence. Cambridge University Press.

Bourgoin, M., Volk, R., Frick, P., Khripchenko, S., Odier, P. \& Pinton, J.-F. 2004 Induction mechanism in von Kármán swirling flows of liquid gallium. Magnetohydrodynamics 40 (1), 13-31.

Brito, D., Cardin, P., Nataf, H.-C. \& Marolleau, G. 1995 Experimental study of a geostrophic vortex of gallium in a transverse magnetic field. Physics of the Earth and Planetary Interiors 91 (1-3), 77-98.

Cortet, P.-P., Chiffaudel, A., Daviaud, F. \& Dubrulle, B. 2010 Experimental evidence of a phase transition in a closed turbulent flow. Physical Review Letters 105 (10), 214501.

Dudley, N.L. \& James, R.W. 1989 Time-dependent kinematic dynamos with stationary flows. Philosophical Transactions of the Royal Society of London. Series A, Mathematical and Physical Sciences 425 (869), 407-429.

Eckert, S., Gerbeth, G., Witke, W. \& Langenbrunner, H. 2001 MHD turbulence measurements in a sodium channel flow exposed to a transverse magnetic field. International Journal of Heat and Fluid Flow 22 (3), 358-364.

Frick, P., Noskov, V., Denisov, S. \& Stepanov, R. 2010 Direct measurement of effective magnetic diffusivity in turbulent flow of liquid sodium. Physical Review Letters 150, 184502.

Gailitis, A., Lielausis, O., Platacis, E., Dement'ev, S., Cifersons, A., Gerbeth, G., Gundrum, T., Stefani, F., Christen, M. \& Will, G. 2001 Magnetic field saturation in the Riga dynamo experiment. Physical Review Letters 86, 3024-3027.

Gallet, B., Berhanu, M. \& Mordant, N. 2009 Influence of an external magnetic field on forced turbulence in a swirling flow of liquid metal. Physics of Fluids 21 (8), 085107.

Kenjeres, S. \& Hanjalic, K. 2009 Tackling complex turbulent flows with transient RANS. Fluid Dynamics Research 41, 012201.

Klein, R. \& Pothérat, A. 2010 Appearance of three dimensionality in wall-bounded MHD flows. Physical Review Letters 104 (3), 034502.

Marié, L. \& Daviaud, F. 2004 Experimental measurement of the scale-by-scale momentum transport budget in a turbulent shear flow. Physics of Fluids 16 (2), 457-461.

MoffatT, H. K. 1961 The amplification of a weak applied magnetic field by turbulence in fluids of moderate conductivity. Journal of Fluid Mechanics 11, 625-635.

Moffatt, H. K. 1978 Magnetic field generation in electrically conducting fluid. Cambridge University Press.

Monchaux, R., Berhanu, M., Aumaître, S., Chiffaudel, A., Daviaud, F., Dubrulle, B., Ravelet, F., Fauve, S., Mordant, N., Pétrélis, F., Bourgoin, M., Odier, P., PinTON, J.-F., Plihon, N. \& Volk, R. 2009 The von Kármán sodium experiment: turbulent dynamical dynamos. Physics of Fluids 21, 035108.

Moreau, R. 1998 MHD turbulence at the laboratory scale: established ideas and new challenges. Applied Scientific Research 58 (1-4), 131-147.

MüCK, B., Günther, C., Müller, U. \& L.ÊBüHler 2000 Three-dimensional MHD flows in rectangular ducts with internal obstacles. Journal of Fluid Mechanics 418, 265-295. 
Müller, U., Stieglitz, R. \& Horanyi, S. 2006 Experiments at a two-scale dynamo test facility. Journal of Fluid Mechanics 552, 419-440.

Odier, P., Pinton, J.-F. \& Fauve, S. 1998 Advection of a magnetic field by a turbulent swirling flow. Physical Review E 58, 7397-7401.

Odier, P., Pinton, J.-F. \& Fauve, S. 2000 Magnetic induction by coherent vortex motion. European Physical Journal B 16, 373-378.

PARker, E. N. 1955 Hydromagnetic dynamo models. Astrophysics Journal 122, 293-314.

Pétrélis, F., Bourgoin, M., Marié, L., Burguete, J., Chiffaudel, A., Daviaud, F., Fauve, S., Odier, P. \& Pinton, J.-F. 2003 Nonlinear magnetic induction by helical motion in a liquid sodium turbulent flow. Physical Review Letters 90 (17), 174501.

Ponomarenko, Yu. B. 1973 Theory of the hydromagnetic generator. Journal of Applied Mechanics and Technologics Physics 14, 775-778.

RAVELET, F. 2005 Bifurcations globales hydrodynamiques et magnétohydrodynamiques dans un écoulement de von Kármán turbulent. (http://tel.archives-ouvertes.fr/tel-00011016/). PhD thesis, École doctorale de l'École Polytechnique.

Ravelet, F., Chiffaudel, A. \& Daviaud, F. 2008 Supercritical transition to turbulence in an inertially driven von Kármán closed flow. Journal of Fluid Mechanics 601, 339-364.

Ravelet, F., Chiffaudel, A., Daviaud, F. \& LÉorat, J. 2005 Toward an experimental von Kármán dynamo: numerical studies for an optimized design. Physics of Fluids 17 (11), 117104

Roberts, G. O. 1972 Dynamo action of fluid motions with two-dimensional periodicity. Philosophical Transactions of the Royal Society of London. Series A, Mathematical and Physical Sciences 271 (1216), 411-454.

Sommeria, J. \& Moreau, M. 1982 Why, how and when, MHD-turbulence becomes twodimensional. Journal of Fluid Mechanics 118, 507-518.

De la Torre, A. \& Burguete, J. 2007 Slow dynamics in a turbulent von Kármán swirling flow. Physical Review Letters 99, 054101.

Volk, R., Odier, P. \& Pinton, J.-F. 2006 Fluctuation of magnetic induction in von Kármán swirling flows. Physics of Fluids 18 (8), 085105.

ZikAnov, O. \& Thess, A. 1998 Direct numerical simulation of forced MHD turbulence at low magnetic Reynolds number. Journal of Fluid Mechanics 358, 299-333. 